\title{
On Reliability of Graphs with Node Failures
}

\author{
Olivier Goldschmidt \\ Department of Mechanical Engineering, The University of Texas at Austin, Austin, \\ Texas 78712-1175 \\ Patrick Jaillet \\ MSIS Department, The University of Texas at Austin, Austin, Texas 78712-1175, \\ and Laboratoire de Mathématiques Appliquées, ENPC, France \\ Richard LaSota* \\ Department of Mechanical Engineering, The University of Texas at Austin, Austin, \\ Texas 78712-1175
}

We consider the reliability of graphs for which nodes fail independently of each other with a constant probability $1-p$. The reliability of a graph is defined to be the probability that the induced subgraph of surviving nodes is connected. A graph is said to be uniformly best when, for all choices of $p$, it is most reliable in the class of graphs with the same number of nodes and same number of edges. In this paper, we first extend the existing known set of uniformly best graphs. Next, we show that most classes of sparse graphs do not contain a uniformly best graph. Finally, we introduce the important notions of locally best and asymptotically best graphs and illustrate these concepts with a detailed study of graphs having the same number of nodes and edges. (c) 1994 John Wiley \& Sons, Inc.

\section{INTRODUCTION}

Reliability theory is concerned mainly with computing the probability that a complex system is functional given the failure probabilities of its elements. Network reliability pertains to systems that can be modeled as graphs whose vertices and/or edges have associated probabilities of being operational. This latter field has been the focus of abundant scientific literature in the last decades. For recent surveys, the reader is referred to the books of Colbourn [4] and Shier [9]. Various reliability measures, linked to different types of system performance, have been defined but most of them suppose that the vertices of the graph are perfectly reliable while its edges fail independently of each other with given probabilities.

\footnotetext{
* The work of this author has been supported by a University Fel-
} lowship.
Only recently have experts in network reliability been interested in graphs whose edges never fail but whose vertices break down independently of each other with given failure probabilities. For many applications, this model is more realistic. For example, in large communication networks, the nodes usually represent complex systems while the links are sturdy cables or wires or, even better, radio waves. Attempts have been made to develop tools to measure the probability that the residual network is connected, i.e., there exists a path using only nonfailed components between any two of its working vertices.

Shier [9] described the two broad objectives of reliability theory as the analysis question of assessing a given system's reliability and the synthesis question of designing the most reliable system from given elements. In our case, with respect to the analysis question, except for special graph structures (see $[2,5]$ ), the problem of computing 
the residual node connectedness is NP-hard [12]. The synthesis problem that we investigate is the following: Given $n$ nodes with failure probability $1-p$ and $m$ perfectly reliable links, construct a graph with $n$ vertices and $m$ edges that maximizes the node reliability function. When $p$ is close to 1 , a most reliable graph will be one with maximum connectivity, $\kappa$ (see [2, pp. 11-12]). Boesch and Felzer [1] showed that regular complete multipartite graphs belong to this class; other results are due to Smith [10], Hakimi and Amin [8], and Doty [6]. On the other hand, if the nodes are very unreliable ( $p$ is close to 0 ), then one wishes to design a graph that maximizes the number of connected induced subgraphs of three vertices. There are already several partial results on this question. A recent $\mathrm{PhD}$ thesis by Stivaros [11] summarized the findings. The complete solution to these questions is far from being known, however, for all possible $n$ and $m$.

A more general question is to ask whether, for given $n$ and $m$, there exists uniformly best graphs, i.e., graphs that are most reliable for all $p, 0<p<1$. As an example, for the class of spanning trees $(m=n-1)$, Stivaros [11] showed that the star graph is uniformly best. If, however, $m=n$, then there are no uniformly best graphs.

In this paper, we first extend the existing known set of uniformly best graphs. Next, we show that most classes of sparse graphs do not contain a uniformly best graph. Finally, we introduce the important notions of locally best and asymptotically best graphs and illustrate these concepts via the detailed study of the case $m=n$. Section 2 contains definitions and two technical lemmas that are used in some of the proofs in the following sections. In [2], Bermond is credited with the result that almost regular complete $k$-partite graphs are uniformly best in their class, though no proof has been published. In Section 3, we present a rigorous proof of this claim. Also, in Section 3, we show that the complete bipartite graphs, $K_{b, b+2}$, are uniformly best. We also show that not every complete $k$ partite graph whose partition sizes differ by at most two is uniformly best and, finally, that $K_{b, b+i}$ is not uniformly best when $i>2$. In Section 4, we establish one of the main results of this paper, namely, that most sparse classes do not contain uniformly best graphs. From this result, it follows that there are at least two locally best graphs in these classes. In the case where the number of vertices equals the number of edges, $n=m$, we prove that there exists exactly two locally best graphs, one that maximizes the number of induced subgraphs on $r$ vertices, $0 \leq r$ $\leq n-2$, and the other having maximum connectivity in its class. In Section 5, we motivate and introduce asymptotically best families of graphs. These families consist of graphs that are most reliable as the number of vertices increase, no matter what $p$ is. Finally, concluding remarks and open problems are presented in Section 6.

\section{DEFINITIONS AND PRELIMINARY RESULTS}

We define $\Omega(n, m)$ to be the set of all connected graphs with $n$ nodes and $m$ edges. Assume that the nodes of a graph fail independently of each other with probability 1 $-p$. The reliability equation gives the probability that a graph $G$ is connected:

$$
R(G)=\sum_{r=1}^{n} S_{r}(G) p^{r}(1-p)^{n-r}
$$

Here, $S_{r}(G)$ is the number of connected induced subgraphs of $G$ that contain exactly $r$ nodes. An $r$-cutset of $G$ is defined to be a set of $r$ nodes of $G$ that, when removed from $G$, leave it disconnected. The number of $r$-cutsets of $G$ is denoted $C_{r}(G)$. Since any set of $r$ nodes in $G$ must either be connected or disconnected, the following relation holds:

$$
S_{r}(G)+C_{n-r}(G)=\left(\begin{array}{l}
n \\
r
\end{array}\right)
$$

Given $p$, there is always at least one locally best graph in $\Omega(n, m)$, i.e., a graph that is most reliable [since $\Omega(n, m)$ is finite]. If there exists $G \in \Omega(n, m)$ that is most reliable for all $p, 0<p<1$, then $G$ is called uniformly best.

A graph $G \in \Omega(n, m)$ is $S_{r}$-optimal if $S_{r}(G) \geq S_{r}(H)$ for all $H \in \Omega(n, m) . \kappa(H)$ denotes the connectivity of a graph $H$. A $\kappa$-optimal graph in $\Omega(n, m)$ will have the highest possible connectivity $(\kappa=\lfloor 2 m / n\rfloor)$ and also the maximum $S_{n-\kappa}$ among all graphs in $\Omega(n, m)$ with connectivity $\kappa$.

A complete almost regular $k$-partite graph is a graph whose vertex set can be partitioned into $k$ independent sets, the sizes of which differ by at most one, and such that any two vertices belonging to different partitions are connected by an edge. We will assume that there are $h$ independent sets of size $b+1$, and $k-h$ independent sets of size $b$. The total number of nodes in this complete almost regular $k$-partite graph is then $n=(k-h) b+h(b$ $+1)=k b+h$. The degree of any node is either $n-b$ or $n-b-1$. By counting the degree of each node, we then have $2 m=(k-h) b(n-b)+h(b+1)(n-b-1)$.

Given a graph $G(V, E)$, the neighbor set of a set $A$ $\subseteq V$ is denoted $N_{A}$ and contains all nodes of $V A$ that are adjacent to a node of $A$.

The following lemma will be used in this paper:

Lemma 1. Let $\left(d_{i}\right)_{i=1}^{n}$ be the degree sequence of a graph $H$ with $n$ nodes, then

$$
C_{r}(H) \geq \frac{1}{n-r} \sum_{i=1}^{n}\left(\begin{array}{c}
n-d_{i}-1 \\
r-d_{i}
\end{array}\right) .
$$


Proof. Let $V$ be the set of nodes of $H$ and let $C_{r}(H)$ $=q$. Let the $q$ r-cutsets of $H$ be denoted $A_{1}, A_{2}, \ldots$, $A_{q}$. Now let $N_{i}$ be the neighbor set of $A_{i}$, i.e., $N_{i}=N_{A_{i}}, 1$ $\leq i \leq q$ :

$$
N_{i} \subseteq V A_{i} \Rightarrow\left|N_{i}\right| \leq n-r,
$$

so

$$
C_{r}(H)(n-r)=q(n-r) \geq \sum_{i=1}^{q}\left|N_{i}\right|
$$

For $x \in V$, let $f(x)$ be the number of $N_{i}$ 's that contain $x$. Then,

$$
\sum_{i=1}^{q}\left|N_{i}\right|=\sum_{x \in V} f(x)
$$

If the degree of node $x$ is $d(x)$, then

$$
f(x) \geq\left(\begin{array}{c}
n-d(x)-1 \\
r-d(x)
\end{array}\right)
$$

because we can find at least $\left(\begin{array}{c}n-d(x)-1 \\ r-d(x)\end{array}\right)$ distinct $r$-cutsets that isolate $x$. The lemma then follows from (5), (6), and (7).

The next lemma helps bound the sum from the last lemma.

Lemma 2. Given integers $n, t, u, b$, and $a_{j}, 1 \leq j \leq n$ with $0 \leq b<n, t \geq 0$, and $\sum_{j=1}^{n} a_{j}=b$, then

$$
\sum_{j=1}^{n}\left(\begin{array}{l}
t+a_{j} \\
u+a_{j}
\end{array}\right) \geq(n-b)\left(\begin{array}{l}
t \\
u
\end{array}\right)+b\left(\begin{array}{l}
t+1 \\
u+1
\end{array}\right) .
$$

Proof. First note that if $a_{1}>0, a_{2} \leq 0$, then

$$
\begin{aligned}
\left(\begin{array}{l}
t+a_{1} \\
u+a_{1}
\end{array}\right)+\left(\begin{array}{l}
t+a_{2} \\
u+a_{2}
\end{array}\right) & \\
& \geq\left(\begin{array}{l}
t+a_{1}-1 \\
u+a_{1}-1
\end{array}\right)+\left(\begin{array}{l}
t+a_{2}+1 \\
u+a_{2}+1
\end{array}\right)
\end{aligned}
$$

This is trivially true for $a_{1}=1$ and $a_{2}=0$, and for $a_{1}$ $-a_{2} \geq 2$, we have

$$
\begin{aligned}
&\left(\begin{array}{l}
t+a_{1} \\
u+a_{1}
\end{array}\right)+\left(\begin{array}{l}
t+a_{2} \\
u+a_{2}
\end{array}\right) \geq\left(\begin{array}{l}
t+a_{1}-1 \\
u+a_{1}-1
\end{array}\right) \\
&+\left(\begin{array}{l}
t+a_{2}+1 \\
u+a_{2}+1
\end{array}\right)+\sum_{i=0}^{a_{1}-a_{2}-2}\left(\begin{array}{c}
t+a_{2}+i \\
u+a_{2}+i+2
\end{array}\right),
\end{aligned}
$$

and the left-hand side will always be greater or equal to the first two terms on the right.

When there exists an $a_{j}>1$ or $a_{j}<0$, use the above inequality to bring the $a_{j}$ closer to 1 and 0 . When all the $a_{j}$ are 0 or 1 , then there are $b$ 1's and $n-b 0$ 's (since $\left.\sum a_{j}=b\right)$ and the lemma follows.

\section{UNIFORMLY BEST GRAPHS}

\subsection{Background}

Stivaros [11] showed that the star graph, $K_{1, n-1}$ is uniformly best in $\Omega(n, n-1)$ by showing that it is $S_{r}$-optimal for all $r$. In fact, it is this method that is used to prove all known instances of uniformly best graphs. Stivaros also showed that a complete graph minus a matching is uniformly best in its class. These latter graphs are a subset of a much larger group, the complete almost regular multipartite graphs, which are also uniformly best. This result is attributed to Bermond [2, p. 13] though no proof has been published. We now present a proof.

Theorem 1 (Bermond). Complete almost regular k-partite graphs are uniformly best in $\Omega(n, m)$.

Proof. The idea of our proof is a generalization of the method used by Boesch and Felzer [1] and also used by Smith [10]. Let $G$ be a complete almost regular $k$-partite graph with $n$ nodes and $m$ edges. To show that $R(G)$ $\geq R(H)$ for all $p, 0 \leq p \leq 1$, and for all $H \in \Omega(n, m)$, we calculate $S_{r}(G)$ to show that it is an upper bound for $S_{r}(H)$ for any $H$ and any $r, 1 \leq r \leq n$. Then, it follows that $R(G) \geq R(H)$ from (1). But $S_{r}(G) \geq S_{r}(H) \Leftrightarrow C_{n-r}(G)$ $\leq C_{n-r}(H)$ from (2). So, in order to show $R(G) \geq R(H)$ we show that $C_{r}(G) \leq C_{r}(H)$ for $0 \leq r \leq n-1$ and any $H \in \Omega(n, m)$.

More specifically, let $G$ be such that $V=V_{1} \cup V_{2}$ $\cup \cdots \cup V_{k}, V_{i} \cap V_{j}=\varnothing \forall i \neq j,\left|V_{1}\right|=\left|V_{2}\right|=\cdots$ $=\left|V_{h}\right|=b+1,\left|V_{h+1}\right|=\left|V_{h+2}\right|=\cdots=\left|V_{k}\right|=b$ for some $h$, and $E=\left\{(w, z) \mid w \in V_{i}, z \in V_{j}, i \neq j\right\}$. Let $H$ be an arbitrary graph in $\Omega(n, m)$.

CASE $1.0 \leq r \leq n-(b+2)$.

In this case, $C_{r}(G)=0$; so, trivially, $C_{r}(G) \leq C_{r}(H)$.

CASE 2. $n-2 \leq r \leq n-1$.

This case is also easy. For $r=n-2, C_{r}(G)=C_{r}(H)$

$=\left(\begin{array}{l}n \\ 2\end{array}\right)-m$, and for $r=n-1, C_{r}(G)=C_{r}(H)=0$.

CASE 3. $n-(b+1) \leq r \leq n-2$.

In this case, $2 \leq n-r \leq b+1$. First we calculate $C_{r}(G)$. For a set to be a $r$-cutset of $G$, all the remaining $n-r$ nodes must lie in the same part of $G$, i.e., the same $V_{i}$. Since there are $k-h$ parts of size $b$ and $h$ parts of size $b+1$, 


$$
C_{r}(G)=(k-h)\left(\begin{array}{c}
b \\
n-r
\end{array}\right)+h\left(\begin{array}{l}
b+1 \\
n-r
\end{array}\right) .
$$

Starting with Lemma 1, we obtain

$$
C_{r}(H) \geq \frac{1}{n-r} \sum_{i=1}^{n}\left(\begin{array}{c}
n-d\left(x_{i}\right)-1 \\
r-d\left(x_{i}\right)
\end{array}\right)
$$

where $x_{i}, i=1 \cdots n$, are the $n$ nodes of $H$. We use Lemma 2 to bound this sum. First, rewrite the sum on the right-hand side of (12):

$$
\begin{aligned}
& \sum_{i=1}^{n}\left(\begin{array}{c}
n-d\left(x_{i}\right)-1 \\
r-d\left(x_{i}\right)
\end{array}\right) \\
& \quad=\sum_{i=1}^{n}\left(\begin{array}{c}
(b-1)+\left(n-b-d\left(x_{i}\right)\right) \\
(r+b-n)+\left(n-b-d\left(x_{i}\right)\right)
\end{array}\right) .
\end{aligned}
$$

To apply the lemma, we let the $a_{i}$ be $n-b-d\left(x_{i}\right)$ :

$$
\begin{aligned}
\sum_{i=1}^{n} a_{i}= & n(n-b)-2 m \\
= & n(n-b)-(k-h) b(n-b) \\
& -h(b+1)(n-b)+h(b+1) \\
= & (n-b)[n-(k-h) b-h(b+1)] \\
& +h(b+1) \\
= & h(b+1),
\end{aligned}
$$

so

$$
\begin{aligned}
\sum_{i=1}^{n}\left(\begin{array}{c}
n-d\left(x_{i}\right)-1 \\
r-d\left(x_{i}\right)
\end{array}\right) & (n-h(b+1))\left(\begin{array}{c}
b-1 \\
r+b-n
\end{array}\right) \\
& +h(b+1)\left(\begin{array}{c}
b \\
r+b-n+1
\end{array}\right) \\
= & (k-h) b\left(\begin{array}{c}
b-1 \\
r+b-n
\end{array}\right) \\
& +h(b+1)\left(\begin{array}{c}
b \\
r+b-n+1
\end{array}\right) \\
= & (k-h) b\left(\begin{array}{c}
b-1 \\
n-r-1
\end{array}\right)+h(b+1)\left(\begin{array}{c}
b \\
n-r-1
\end{array}\right) \\
= & (k-h)\left(\begin{array}{c}
b \\
n-r
\end{array}\right)(n-r)+h\left(\begin{array}{l}
b+1 \\
n-r
\end{array}\right)(n-r) \\
= & C_{r}(G)(n-r) .
\end{aligned}
$$

Together with (12), this gives $C_{r}(H) \geq C_{r}(G)$ as desired.
In conclusion, $C_{r}(H) \geq C_{r}(G)$ for $0 \leq r \leq n-1$, which implies that $G$ is uniformly best in $\Omega(n, m)$.

\subsection{Extensions}

In this subsection, we investigate possible extensions of the result obtained above. Let $K_{b, b+2}$ denote the complete bipartite graph with a node partition of cardinalities $b$ and $b+2$, respectively, and let $\Omega\left(2 b+2, b^{2}+2 b\right)$ be the set of all connected graphs with $n=2 b+2$ nodes and $m$ $=b^{2}+2 b$ edges. The main result of this subsection is the following:

Theorem 2. The complete bipartite graph $K_{b, b+2}$ is uniformly best among all graphs in $\Omega\left(2 b+2, b^{2}+2 b\right)$, $b \geq 1$.

Proof. For all graphs $G$ in $\Omega\left(2 b+2, b^{2}+2 b\right)$, let $C_{r}(G)$ be the number of node cutsets of $G$ of cardinality $r$. We will prove this lemma by showing that for all graphs $G$ in $\Omega\left(2 b+2, b^{2}+2 b\right)$ we have $C_{r}\left(K_{b, b+2}\right) \leq C_{r}(G)$, for all 0 $\leq r \leq n$.

First, note that for any graph $G \in \Omega(n, m)$ we have $C_{0}(G)=0, C_{n}(G)=1, C_{n-1}(G)=0$, and $C_{n-2}(G)=\left(\begin{array}{c}n \\ 2\end{array}\right)$ $-m$. Also, if $k$ denotes the connectivity of the graph $G$, then $C_{r}(G)=0$ for $r<\kappa$.

The connectivity of the complete bipartite graph $K_{b, b+2}$ is easily seen to be $b$ [which is maximum in $\Omega\left(2 b+2, b^{2}\right.$ $+2 b)$ ], and a simple counting argument shows that we have

$$
C_{r}\left(k_{b, b+2}\right)=\left(\begin{array}{l}
b+2 \\
r-b
\end{array}\right)+\left(\begin{array}{c}
b \\
r-b-2
\end{array}\right)
$$

for all $b \leq r \leq 2 b-1$, with the convention that $\left(\begin{array}{l}x \\ y\end{array}\right)=0$ if $y<0$.

Let us then bound $C_{r}(G), b \leq r \leq 2 b-1$, for any graph $G$. Note that if $G \in \Omega\left(2 b+2, b^{2}+2 b\right)$ then either $G$ has at least one node of degree $b$ or else it must have at least one node of degree $\leq b-1$ (indeed, otherwise the sum of the degree of the nodes of $G$ would exceed $2 m$ ). Let us consider both cases separately.

CASE 1. $G$ has at least one node of degree $b$.

Let $v$ be such a node, and let $G-v$ be the induced subgraph of $G$ obtained by removing node $v$. We then have

$$
\begin{aligned}
C_{r}(G) & \geq\left(\begin{array}{c}
n-1-b \\
r-b
\end{array}\right)+C_{r-1}(G-v) \\
& =\left(\begin{array}{c}
b+1 \\
r-b
\end{array}\right)+C_{r-1}(G-v) .
\end{aligned}
$$

$\left[\left(\begin{array}{c}n-1-b \\ r-b\end{array}\right)\right.$ is a lower bound on the number of cutsets of 
cardinality $r$ that do not include the node $v$.] Now, $G$ $-v$ is in $\Omega\left(2 b+1, b^{2}+b\right)$, a class that contains $K_{b, b+1}$, which we know, from Subsection 3.1 (see Theorem 1), to be uniformly best. So, we have

$$
\begin{aligned}
& C_{r-1}(G-v) \geq C_{r-1}\left(K_{b, b+1}\right) \\
&=\left(\begin{array}{c}
b+1 \\
r-b-1
\end{array}\right)+\left(\begin{array}{c}
b \\
r-b-2
\end{array}\right),
\end{aligned}
$$

the last equality being obtained again via a simple counting argument. From (17) and (18), we then have

$$
\begin{aligned}
C_{r}(G) & \geq\left(\begin{array}{l}
b+1 \\
r-b
\end{array}\right)+\left(\begin{array}{c}
b+1 \\
r-b-1
\end{array}\right)+\left(\begin{array}{c}
b \\
r-b-2
\end{array}\right) \\
& =\left(\begin{array}{l}
b+2 \\
r-b
\end{array}\right)+\left(\begin{array}{c}
b \\
r-b-2
\end{array}\right) .
\end{aligned}
$$

One then concludes Case 1 from (16) and (19).

CASE 2. $G$ has no nodes of degree $b$, and so has at least one node of degree $\leq b-1$. Let $d^{*}$ be the degree of a node of $G$ not exceeding $b-1$, and let $w$ be a node of $G$ of degree $d^{*}$. Then, using the same rationale as for (17), we have

$$
C_{r}(G) \geq\left(\begin{array}{c}
2 b+1-d^{*} \\
r-d^{*}
\end{array}\right)+C_{r-1}(G-w) .
$$

$G-w$ belongs to $\Omega\left(2 b+1, b^{2}+2 b-d^{*}\right)$, so let $n^{\prime}$ $=2 b+1$ and $m^{\prime}=b^{2}+2 b-d^{*}$. By using the bounding method given in Lemma 1 of Section 2, we have

$$
\begin{aligned}
& C_{r-1}(G-w) \\
& \geq \frac{1}{n^{\prime}-r+1} \sum_{j=1}^{n^{\prime}}\left(\begin{array}{l}
n^{\prime}-1-d\left(v_{j}\right) \\
r-1-d\left(v_{j}\right)
\end{array}\right) \\
& =\frac{1}{2 b+2-r} \sum_{v \in G-w}\left(\begin{array}{c}
2 b-d\left(v_{j}\right) \\
r-1-d\left(v_{j}\right)
\end{array}\right) .
\end{aligned}
$$

Let us now apply Lemma 2 of Section 2 in order to bound this last summation. We need to consider two subcases, depending on $d^{*}$.

(i) $\lceil(b-1) / 2\rceil \leq d^{*} \leq b-1$ : We use Lemma 2 with $a_{j}=b+1-d\left(v_{j}\right)$. Using (20) and (21), we then get

$$
\begin{aligned}
C_{r}(G) \geq & \left(\begin{array}{c}
2 b+1-d^{*} \\
r-d^{*}
\end{array}\right) \\
& +\frac{1}{2 b+2-r}\left(\left(3 b-2 d^{*}\right)\left(\begin{array}{c}
b-1 \\
r-b-2
\end{array}\right)\right.
\end{aligned}
$$

$$
\left.+\left(2 d^{*}+1-b\right)\left(\begin{array}{c}
b \\
r-b-1
\end{array}\right)\right)
$$

(ii) $1 \leq d^{*} \leq\lfloor(b-1) / 2\rfloor$ : We use Lemma 2 with $a_{j}$ $=b+2-d\left(v_{j}\right)$, and finally get

$$
\begin{gathered}
C_{r}(G) \geq\left(\begin{array}{c}
2 b+1-d^{*} \\
r-d^{*}
\end{array}\right)+\frac{1}{2 b+2-r} \\
\times\left(\begin{array}{c}
\left(b-1-2 d^{*}\right)\left(\begin{array}{c}
b-2 \\
r-b-3
\end{array}\right) \\
\left.+\left(2 d^{*}+2+b\right)\left(\begin{array}{c}
b-1 \\
r-b-2
\end{array}\right)\right)
\end{array}\right.
\end{gathered}
$$

Now, for fixed $b$ and $r$, the lower bounds are, in both subcases, decreasing functions of $d^{*}$. Moreover, the lower bound in subcase (ii) is always larger than the lower bound in subcase (i). We can then concentrate on our worst case, i.e., when $d^{*}=b-1$. In that case, we have

$$
\begin{aligned}
& C_{r}(G) \geq\left(\begin{array}{c}
b+2 \\
r-b+1
\end{array}\right) \\
& +\frac{1}{2 b+2-r}\left((b+2)\left(\begin{array}{c}
b-1 \\
r-b-2
\end{array}\right)\right. \\
& \left.+(b-1)\left(\begin{array}{c}
b \\
r-b-1
\end{array}\right)\right) \\
& =\left(\begin{array}{l}
b+2 \\
r-b
\end{array}\right)+\left(\begin{array}{c}
b \\
r-b-2
\end{array}\right)+\left(\begin{array}{c}
b+1 \\
r-b+1
\end{array}\right) \\
& -\frac{2}{2 b+2-r}\left(\begin{array}{c}
b-1 \\
r-b-1
\end{array}\right) \\
& \geq\left(\begin{array}{l}
b+2 \\
r-b
\end{array}\right)+\left(\begin{array}{c}
b \\
r-b-2
\end{array}\right) \text {. }
\end{aligned}
$$

One then concludes Case 2 from (16) and (24).

Remarks. The previous result is best possible in the following two senses:

1. $K_{b, b+i}$ is not uniformly best for $i>2$. Indeed, its connectivity is $b$, and thus it is not of maximum connectivity in $\Omega\left(2 b+i, b^{2}+i b\right)$ (the maximum connectivity is $\lfloor 2 m / n\rfloor=\left\lfloor 2\left(b^{2}+i b\right) /(2 b+i)\right\rfloor>b$ when $\left.i>2\right)$. Moreover, $K_{b, b+i}$ is not $S_{k}$-optimal for a large number of $k$. For instance, we have constructed a graph $G$ $\in \Omega\left(2 b+3, b^{2}+3 b\right)$ such that $C_{r}(G)<C_{r}\left(K_{b, b+3}\right)$ for any $r$ in the range $b+1 \leq r \leq 1.18 b$ (see [7]).

2. The complete "almost-almost" regular $k$-partite graphs are not (in general) uniformly best. $H=(V, E)$ is said 
to be a complete almost-almost regular $k$-partite graph if $V$ is partitioned in $k$ sets, $V_{1}, \ldots, V_{k}$, where $\left|V_{1}\right|$ $=\cdots=\left|V_{h}\right|=b+2,\left|V_{h+1}\right|=\cdots=\left|V_{k}\right|=b$, with $0<h<k$ and $b>0$, and if $E=\left\{(v, w): v \in V_{i}\right.$, $\left.w \in V_{j}, i \neq j\right\}$.

$H$ is then a member of $\Omega(n, m)$ with $n=h(b+2)$ $+(k-h) b$ and $2 m=(n-b)(k-h) b+(n-b-2) h(b$ +2 ). To prove our claim, it suffices to show that $H$ is not always $\kappa$-optimal. The maximum connectivity of any graph $G \in \Omega(n, m)$, say $\kappa^{*}$, easily verifies $(n-b$ $-2) \leq \kappa^{*} \leq(n-b)$. Now the connectivity of $H$ is $n$ $-b-2$. We just have to find values of $b, h$, and $k$ for which $k^{*}=n-b-1$. After some elementary steps, one can show that this will be the case whenever we have $b=2 h /(k-2 h)$. As an example, take $h=1$ and $k=4$, then, $b=1$ and $H$ is in $\Omega(6,12)$; the maximum connectivity is then $24 / 6=4$, but the connectivity of $H$ is 3 .

In conclusion, the result of Subsection 3.1 is best possible and the uniformly best property of $K_{b, b+2}$ is an exception.

\section{LOCALLY BEST GRAPHS}

\subsection{Background}

We do not know if there are any other classes other than those of Section 3 that have uniformly best graphs. However, we show next that for a wide range of the sparse classes $\left(m<n^{2} / 4, m \neq n-1\right)$, uniformly best graphs do not exist. There must be at least two locally best graphs in these classes, and, in fact, we show that there are exactly two in the class $\Omega(n, n)$.

\subsection{Many Classes Do Not Have Uniformly Best Graphs}

A uniformly best graph necessarily is both $S_{3}$-optimal and $\kappa$-optimal [2]. Stivaros [11] showed that the star graph with one extra edge is the unique $S_{3}$-optimal graph in its class, whereas the cycle of length $n$ is the unique $k$-optimal graph. Hence, no uniformly best graph exists in $\Omega(n, n)$.

This same idea can be used to show that most sparse classes do not contain uniformly best graphs. Specifically, given a class $\Omega(n, m)$, first find a graph in it with a relatively large value for $S_{3}$. Since Stivaros [11] showed that complete bipartite graphs are 3-optimal in their classes, we choose a complete bipartite graph if possible, and if not, then a complete bipartite plus or minus some edges. Then, second, we use the fact that the minimum degree of a $\kappa$ optimal graph in $\Omega(n, m)$ will be $d=\lfloor 2 m / n\rfloor$ and find an upper bound for the $S_{3}$ value of any $k$-optimal graph. The result for most sparse classes will be that all $\kappa$-optimal graphs in $\Omega(n, m)$ cannot also be 3-optimal.
Theorem 3. For $m=r n+k, 2 \leq r \leq n / 5-4,0 \leq k$ $<n / 2$, then $\Omega(n, m)$ does not contain a uniformly best graph.

To prove this result, we will use two lemmas. The first lemma shows the existence of a graph with a fairly high value of $S_{3}$, namely, a bipartite graph with extra edges added.

Lemma 3. If $m=r n+k, m \leq\left(\begin{array}{c}n \\ 2\end{array}\right)$, and $0 \leq k<n$, then $\exists G \in \Omega(n, m)$ with $S_{3}(G) \geq r(n-r)(n-2) / 2$.

Proof. Let $J=K_{r, n-r}$. From Stivaros' formula for $S_{3}$ [11, Chap. 1], we know that

$$
S_{3}(J)=\sum_{i=1}^{n}\left(\begin{array}{l}
d_{i} \\
2
\end{array}\right)-2 \tau(J)
$$

where $\tau(J)$ is the number of triangles of $J$. But $\tau(J)=0$ since $J$ is bipartite. Hence,

$$
\begin{aligned}
S_{3}(J) & =\sum_{i=1}^{n}\left(\begin{array}{l}
d_{i} \\
2
\end{array}\right)=r\left(\begin{array}{c}
n-r \\
2
\end{array}\right)+(n-r)\left(\begin{array}{l}
r \\
2
\end{array}\right) \\
& =\frac{r(n-r)(n-2)}{2} .
\end{aligned}
$$

$J$ has $r n-r^{2}$ edges. Add any $r^{2}+k$ edges to obtain $G$ $\in \Omega(n, m=r n+k)$. Then,

$$
S_{3}(G) \geq S_{3}(J)=\frac{r(n-r)(n-2)}{2} .
$$

If $\left(d_{i}\right)_{i=1}^{n}$ is the degree sequence of a graph, then the next lemma will bound the function $\sum_{i=1}^{n}\left(\frac{d_{i}}{2}\right)$.

Lemma 4. Let $\left(d_{i}\right)_{i}$ be the degree sequence of a graph of $\Omega(n, m)$, so that $\sum_{=1}^{n} d_{i}=2 m$. Also, let $d=\lfloor 2 m / n\rfloor$ and $d$ $\leq d_{i} \leq n-1$ for $i=1 \cdots n$. Then,

$$
\sum_{i=1}^{n}\left(\begin{array}{l}
d_{i} \\
2
\end{array}\right) \leq(n-2)\left(\begin{array}{l}
d \\
2
\end{array}\right)+\left(\begin{array}{c}
2 d \\
2
\end{array}\right)+\left(\begin{array}{c}
n-1 \\
2
\end{array}\right) .
$$

Proof. Assume that the $d_{i}$ are arranged in nondecreasing order. $2 m \geq n d$ since $d_{i} \geq d$ for all $i$, so let $2 m=n d$ $+p$ with $p \geq 0$. Since $d=\lfloor 2 m / n\rfloor=\lfloor(n d+p) / n\rfloor=\lfloor d$ $+p / n\rfloor, p$ must be less than $n: p \leq n-1$. For any $d_{i}>d$ where $1 \leq i \leq n-2$, decreasing $d_{i}$ by 1 and increasing $d_{n}$ (or $d_{n-1}$ ) by 1 will only increase the value of $\sum_{i=1}^{n}\left(\begin{array}{c}d_{i} \\ 2\end{array}\right)$. We continue in this way until $d_{i}=d$ for $i=1 \cdots n-1$, if possible, in which case $d_{n}=d+p$ (or if $d+p>n$ -1 , then until $d_{n}=n-1$ and $d_{n-1}=p+2 d+1-n$ $\leq 2 d$ ). In either case, the result follows. 
Proof of Theorem 3. Let $H \in \Omega(n, m)$ with maximum connectivity, i.e., $\kappa(H)=\lfloor 2 m / n\rfloor$, where $\kappa(H)$ is the node connectivity of the graph $H$. We have

$$
\begin{aligned}
d & =\kappa(H)=\lfloor 2 m / n\rfloor=\left[\frac{2 m+2 k}{n}\right] \\
& =\left[2 r+\frac{2 k}{n}\right]=2 r .
\end{aligned}
$$

In light of Lemma 3, we need to show that $S_{3}(H)<r(n$ $-r)(n-2) / 2$. We use Lemma 4 to obtain

$$
\begin{aligned}
S_{3}(H) & \leq \sum_{i=1}^{n}\left(\begin{array}{c}
d_{i} \\
2
\end{array}\right) \\
& \leq(n-2)\left(\begin{array}{c}
2 r \\
2
\end{array}\right)+\left(\begin{array}{c}
4 r \\
2
\end{array}\right)+\left(\begin{array}{c}
n-1 \\
2
\end{array}\right) .
\end{aligned}
$$

Then, the question becomes, when is

$$
(n-2)\left(\begin{array}{c}
2 r \\
2
\end{array}\right)+\left(\begin{array}{c}
4 r \\
2
\end{array}\right)+\left(\begin{array}{c}
n-1 \\
2
\end{array}\right) \stackrel{?}{<} \frac{r(n-r)(n-2)}{2}
$$

A little algebra shows that (31) holds given the conditions of the theorem.

Remarks. The above theorem can be refined to handle all classes $\Omega(n, m)$ with $n \leq m<n^{2} / 4-3 n$ :

1. To handle the case $n / 2 \leq k<n$ in Theorem $3, d$ in (29) becomes $2 r+1$ and a similar result is obtained.

2. To handle the case $n / 5-4 \leq r<n / 4-3$, Lemma 3 must be more sophisticated. Instead of $J=K_{r, n-r}$, let $J=K_{r^{\prime}, n-r^{\prime}}$, where $r^{\prime}$ is the largest integer such that $r^{\prime}(n$ $\left.-r^{\prime}\right) \leq m$.

3. To handle $r=1$, Lemma 3 must be strengthened to consider the increase in $S_{3}(J)$ that results from adding $k+1$ edges.

4. Special cases for low values of $n$ that do not fall into any of the above categories are handled on a case by case basis by a combination of the above techniques. We have used a computer program to verify all cases $\Omega(n, m)$ with $n \leq m<n^{2} / 4-n / 2-2$ where $n \leq 170$.

For details, see [7]. The above technique is more difficult to apply in the range $n^{2} / 4-3 n \leq m<n^{2} / 4-2$ and not all cases have been proved in this range. But for $\Omega\left(2 b+i, b^{2}+i b\right), i \geq 3$, no uniformly best graphs exist. This is because the unique $S_{3}$-optimal graphs in these classes are $K_{b, b+i}$ [11], which are not $\kappa$-optimal.

\subsection{The Locally Best Graphs of $\Omega(n, n)$}

In this subsection, we show that when $n \geq 6$, the star plus one edge and the cycle are the only two locally best graphs of $\Omega(n, n)$. More precisely, we have

Theorem 4. Let $n \geq 6$. The star plus one edge, $K_{1, n-1}$ +1 , is such that, for $3 \leq r \leq n-2$

$$
\begin{aligned}
& S_{r}\left(K_{1, n-1}+1\right)>S_{r}(G) \quad \text { for all } \\
& \qquad G \in \Omega(n, n) \backslash\left\{K_{1, n-1}+1\right\} .
\end{aligned}
$$

The cycle, Cycle, is such that

$$
\begin{aligned}
& S_{n-1}\left(\text { Cycle }_{n}\right)>S_{n-1}(G) \quad \text { for all } \\
& \qquad G \in \Omega(n, n) \backslash\left\{\text { Cycle }_{n}\right\} .
\end{aligned}
$$

Proof. First (33) is true since the cycle is the only graph of $\Omega(n, n)$ of maximum connectivity 2 . In particular, we have $S_{n-1}\left(\right.$ Cycle $\left._{n}\right)=n$ and $S_{n-1}\left(K_{1, n-1}+1\right)=n-1$. Let us now concentrate on (32).

It is easy to see that we have

$$
S_{r}\left(K_{1, n-1}+1\right)=\left(\begin{array}{l}
n-1 \\
r-1
\end{array}\right) \text { for all } 3 \leq r \leq n-2
$$

Moreover, we have $S_{r}\left(\right.$ Cycle $\left._{n}\right)=n$ for all $3 \leq r \leq n-2$, which is clearly less than the values given in (34). More generally, let us prove that for all other graphs $G \in \Omega(n$, $n$ ) we also have

$$
S_{r}(G)<\left(\begin{array}{l}
n-1 \\
r-1
\end{array}\right) \text { for all } 3 \leq r \leq n-2 .
$$

We will do that by induction on $n$. By complete enumeration [there are 13 graphs in $\Omega(6,6)$ ], (35) is easily seen to be true for $n=6$. Let us then assume that (35) is true up to $n-1$, and let us prove it for $n$. Let $G$ be any graph of $\Omega(n, n)$ that is not the cycle Cycle $_{n}$. It has at least a node of degree 1 , say $v$. We then have

$$
\begin{aligned}
& S_{r}(G)=S_{r}(G-v)+S_{r}(G ; v) \text { for all } \\
& \qquad 3 \leq r \leq n-2,
\end{aligned}
$$

where $G-v$ is the induced subgraph of $G$ obtained by removing node $v$, and $S_{r}(G ; v)$ is the total number of connected induced subgraphs of $r$ nodes containing $v$. It is easy to see that one can always choose $v$ such that $G$ $-v$ is not $K_{1, n-2}+1$. By our induction hypothesis, we then know that 


$$
S_{r}(G-v)<\left(\begin{array}{l}
n-2 \\
r-1
\end{array}\right) \text { for all } 3 \leq r \leq n-3
$$

Also, an induced subgraph of $r$ nodes containing $v$ must also contain its unique adjacent node, in order to be connected. We then have the freedom to choose $r-2$ other nodes among the remaining $n-2$ nodes. Hence,

$$
S_{r}(G ; v) \leq\left(\begin{array}{l}
n-2 \\
r-2
\end{array}\right) \text { for all } 3 \leq r \leq n-3 .
$$

From (37) and (38), we then have

$$
\begin{aligned}
S_{r}(G) & <\left(\begin{array}{l}
n-2 \\
r-1
\end{array}\right)+\left(\begin{array}{l}
n-2 \\
r-2
\end{array}\right) \\
& =\left(\begin{array}{l}
n-1 \\
r-1
\end{array}\right) \text { for all } 3 \leq r \leq n-3
\end{aligned}
$$

It remains to cover the case $r=n-2$ in order to complete the proof. If $G-v$ is not the cycle $C y c l e_{n-1}$, then the previous argument still holds. If $G-v$ is the cycle Cycle $_{n-1}$, then the right-hand side of (37) is $n-1$ instead of $n-2$, but now $S_{r}(G ; v)=n-3$, and so we still have $n-1+n-3=2 n-4<\left(\begin{array}{c}n-1 \\ 2\end{array}\right)$ when $n \geq 6$.

From this theorem, it is easy to deduce the following result:

Corollary 1. The star plus one edge and the cycle are the only two locally best graphs in $\Omega(n, n), n \geq 6$.

Remarks. When $n \leq 4$, the cycle is uniformly best, so it is the only locally best graph in $\Omega(n, n)$. When $n=5$, three graphs are locally best: the star plus one edge and the cycle, but also a third graph that has the same reliability as the star plus one edge. This last graph is built from the cycle on four nodes with a leaf attached to one of them.

\section{ASYMPTOTICALLY BEST GRAPHS}

When $\Omega(n, m)$ does not contain uniformly best graphs, there may still be graphs in $\Omega(n, m)$ that are most reliable when $n$ is large enough. For example, for all $0<p \leq 0.99$, the star plus one edge, $K_{1, n-1}+1$, is the most reliable graph in $\Omega(n, n)$, for all $n>16$. This most practical observation motivates the introduction of asymptotically best graphs that intuitively describes graphs that are most reliable as the number of nodes increases, no matter what $p$ is. The precise definition of this notion is as follows:

A family of graphs $\left(G_{n}\right)_{n=1}^{\infty}$, where $G_{n} \in \Omega(n, m(n))$ and $m(n)$ a nondecreasing integer-valued function, is said to be asymptotically best in $(\Omega(n, m(n)))_{n}$ if for all $0<p<1$ there exists an $N(p)$, such that for all $n \geq N(p)$ we have $R\left(G_{n}\right) \geq R(H)$ for all $H$ $\in \Omega(n, m(n))$.

From Theorem 4, it is easy to see that we have

Corollary 2. The family of the star plus one edge, $\left(K_{1, n-1}\right.$ $+1)_{n}$, is asymptotically best in $(\Omega(n, n))_{n}$.

Indeed, we know that for any fixed $n>5, K_{1, n-1}+1$ and $\mathrm{CyCle}_{n}$ are the only two possible best graphs. Also, since $K_{1, n-1}+1$ dominates all $S_{k}$ except $S_{n-1}$, the result follows. Alternatively, one obtains from Theorem 4 that

$$
\begin{aligned}
& R\left(K_{1, n-1}+1\right) \\
& \quad=p+p^{2}(1-p)^{n-2}+(n-1) p(1-p)^{n-1}
\end{aligned}
$$

and that

$$
R\left(\text { Cycle }_{n}\right)=p^{n}+n p(1-p) \frac{(1-p)^{n-1}-p^{n-1}}{1-2 p},
$$

from which it is straightforward to conclude.

\section{CONCLUSION}

In this paper, we proved that most classes of sparse graphs contain no uniformly best graphs. We also extended the set of classes for which there exist uniformly best graphs. The notion of asymptotically best graphs is introduced and illustrated in the class $\Omega(n, n)$.

However, the subject is far from being exhausted. First, there are still classes for which it is not known whether uniformly best graphs exist or not. Among sparse graphs, these are the cases $n^{2} / 4-3 n<m \leq n^{2} / 4-2$. Among dense graphs $\left(m>n^{2} / 4\right)$, these are the cases where $\Omega(n$, $m$ ) does not contain a complete almost regular $k$-partite graph. Also, all uniformly best graphs that have been found so far are $S_{r}$-optimal for all $r$. From (1), this is a sufficient condition for a graph to be uniformly best but it is not known whether the condition is also necessary.

Second, for the classes with no uniformly best graph, one wishes to identify a minimum set of locally best graphs covering the whole range of $p, 0<p<1$. Two related questions are whether the cardinality of such a set is bounded by a constant and whether it is true that if a graph is locally best for two different values of $p$, say $p_{1}$ and $p_{2}$, then it is also locally best for all intermediate values of $p, p_{1} \leq p \leq p_{2}$.

Asymptotically best graphs provide a family of graphs of high reliability. We would like to characterize the functions $m(n)$ that give rise to such families. Finally, we rec- 
ognize the need to generalize these results to the case of graphs with edge weights, so that the problem becomes the design of a graph of maximum reliability with a given cost.

\section{REFERENCES}

[1] F. Boesch and A. Felzer, On the invulnerability of the regular complete $k$-partite graphs. SIAM J. Appl. Math., 20 (1971) 176-182.

[2] F. Boesch, A. Satyanarayana, and C. Suffel, On residual connectedness network reliability. Preprint, Stevens Institute of Technology (1990). Presented at ORSA/TIMS.

[3] B. Bollobas, Random Graphs. Academic Press, London (1985).

[4] C. Colbourn, The Combinatories of Network Reliability. Oxford University Press, New York (1987).

[5] C. Colbourn, A. Satyanarayana, C. Suffel, and K. Sutner, Computing residual connectedness reliability for restricted networks. Disc. Appl. Math. 44 (1993) 221-232.
[6] L. Doty, Extremal connectivity and vulnerability in graphs. Networks 19 (1989) 73-78.

[7] O. Goldschmidt, P. Jaillet, and R. LaSota, Node reliability results. Joint technical Report of the Dept. of Mechanical Engineering and the MSIS Dept., University of Texas at Austin (1992)

[8] S. Hakimi and A. Amin, On the design of reliable networks. Networks 3 (1973) 241-260.

[9] D. R. Shier, Network Reliability and Algebraic Structures. Clarendon Press, Oxford (1991).

[10] D. Smith, Graphs with the smallest number of minimum cut-sets. Networks 14 (1984) 47-62.

[11] C. Stivaros, On the residual node connectedness network reliability model. PhD Dissertation, Dept. of Electrical Engineering and Computer Science, Stevens Institute of Technology (1990).

[12] K. Sutner, A. Satyanarayana, and C. Suffel, The complexity of the residual node connectedness reliability problem. SIAM J. Comput. 20 (1991) 149-155.

Received May 1992

Accepted December 1993 